A6

doi: 10.14232/ syrpharmacognosy.2021.a6

\title{
Study on peroxynitrite-induced oxidation of hydrocurcumins
}

\author{
Gábor Girst
}

Email: girst.gabor@pharmacognosy.hu

Hydrocurcumins (tetrahydrocurcumin, hexahydrocurcumin and octahydrocurcumin) are major phase I, bioreduced metabolites of curcumin, the main bioactive compounds of Turmeric (Curcuma longa). These compounds exhibit similar or, in some cases, even better bioactivities in vitro and in vivo compared to curcumin itself (e.g. antioxidant, anti-inflammatory or cardiovascular protective activity) [1]. Further, unlike curcumin, hydrocurcumins are much more stable in the human body, have a slower metabolism, and better bioavailability.

The reaction between antioxidants and scavenging reactive oxygen or nitrogen species often results in chemically stable, bioactive metabolites. These new metabolites could have altered bioactivity compared to the parent compound, and this activity can be directly translated from oxidative stress. It is reasonable to extend the chemical space by utilizing RONS-mediated oxidation $[2,3]$.

In this work we aimed to study the reaction between peroxynitrite, a biorelevant reactive nitrogen species, and hydrocurcumins to extend the chemical space, and to better understand the mechanism of such antioxidant activity. So far, we isolated 14 different compounds from the reaction mixtures by using RP-HPLC. Among these compounds 4 have been confirmed as fragments of the starting materials.

Supervisor: Attila Hunyadi

\section{Acknowledgements:}

This work was supported by the National Research, Development and Innovation Office, Hungary (NKFIH; K-134704), the Ministry of Human Capacities, Hungary grant 20391-3/2018/FEKUSTRAT and the EFOP 3.6.3-VEKOP-16-2017-00009 project.

\section{References}

[1] Somparn P, et al. Biological \& pharmaceutical bulletin. 2007;30(1):74-8.

[2] Hunyadi A. Medicinal Research Reviews. 2019;39(6):2505-33.

[3] Fási L, et al. Biomolecules. 2020;10(11):1537 